\title{
Divergence Between Adolescent and Parental Perceptions of Conflict in Relationship to Adolescent Empathy Development
}

\author{
Caspar J. Van Lissa $\cdot$ Skyler T. Hawk \\ Susan J. T. Branje $\cdot$ Hans M. Koot • \\ Pol A. C. Van Lier $\cdot$ Wim H. J. Meeus
}

Received: 5 May 2014/Accepted: 3 July 2014/Published online: 11 July 2014

(C) Springer Science+Business Media New York 2014

\begin{abstract}
Adolescents' developing empathy may be associated with the frequency of conflict with parents, as well as the level of agreement between adolescent and parental perceptions of the frequency of such conflicts. This 6-year longitudinal study investigated the link between adolescent empathy development and perceptions of the frequency of parent-child conflict, as reported by 467 adolescents (43\% female, from age 13) and both parents. First, we investigated heterogeneity in empathy development by identifying classes of individuals with similar developmental trajectories. Adolescents were categorized into high-, average-, and low-empathy classes. Initial differences between these classes further increased from age 13 to 16 , particularly for cognitive empathy. To assess the
\end{abstract}

C. J. Van Lissa $(\bowtie) \cdot$ S. T. Hawk · S. J. T. Branje

W. H. J. Meeus

Adolescent Development, Utrecht University, Heidelberglaan 1, 3584 CS Utrecht, The Netherlands

e-mail: c.j.vanlissa@uu.nl; SecretariaatPedagogiek@uu.nl

S. T. Hawk

e-mail: s.t.hawk@cuhk.edu.hk

S. J. T. Branje

e-mail: s.branje@uu.nl

W. H. J. Meeus

e-mail:w.meeus@uu.nl

H. M. Koot - P. A. C. Van Lier

Developmental Psychology, VU University, Van der

Boechorststraat 1, 1081 BT Amsterdam, The Netherlands

e-mail: j.m.koot@vu.nl

P. A. C. Van Lier

e-mail: pac.van.lier@vu.nl

W. H. J. Meeus

Developmental Psychology, Tilburg University, Tilburg,

The Netherlands association between empathy and the frequency of conflict, we compared these empathy classes in terms of initial levels and over-time changes in the frequency of adolescent- and parent-reported conflict. Compared to the average- and high-empathy classes, the low-empathy class evidenced elevated conflict throughout adolescence. Furthermore, the low- and average-empathy classes demonstrated temporary divergence between adolescent- and parent-reported conflict from early- to mid-adolescence, with adolescents underreporting conflict compared to both parents. Adolescents' agreement with parents was moderated by empathy class, while parents were always in agreement with one another. This may suggest that these discrepancies are related to distortions in adolescents' perceptions, as opposed to biased parental reports. These findings highlight the potential importance of early detection and intervention in empathy deficiencies, and suggest that lower adolescent empathy may indicate elevated family conflict, even if a failure to consider parents' perspective leads adolescents to underreport it.

Keywords Empathy · Conflict · Adolescence .

Reporter discrepancies · Longitudinal

\section{Introduction}

During adolescence, parents and children do not always see eye to eye. Adolescent-parent conflict often occurs as youths strive for greater independence (Branje et al. 2013). The frequency of conflict subsides from early to late adolescence (Laursen et al. 1998), which coincides with increasing adolescent empathy (Davis and Franzoi 1991). Three fundamental issues remain unexplored, however. Although researchers have suggested that adolescence is a 
developmentally sensitive period for empathy (Choudhury et al. 2006), longitudinal studies have only examined group-level change (e.g., Eisenberg et al. 2005). Because developmentally sensitive periods are often characterized by further differentiation (Caspi and Moffitt 1991), a person-centered approach might reveal different trajectories of empathy development. The second issue concerns links between empathy development and adolescent-parent conflict. Empathy promotes positive conflict resolution in adolescent friendships (De Wied et al. 2007), and inhibits aggression in adults (Richardson et al. 1994). This suggests that adolescent empathy might also be related to conflict with parents. Third, adolescent and parental perceptions of their relationships often differ, including conflict perceptions (De Los Reyes and Kazdin 2005). Because empathy involves taking others' perspectives and responding considerately to their emotions (Davis 1983), reports of highempathy adolescents should be more in line with those of their parents. Conversely, reports of low-empathy youths might deviate more strongly from parents'. We addressed these issues in a 6-year longitudinal study, by investigating the link between developmental trajectories of adolescents' empathy and the frequency of conflict as reported by adolescents and both parents.

\section{Empathy and the Frequency of Conflict}

Even though many theorists now agree that adolescence is not the turbulent period of "storm and stress" it was once thought to be, some adolescent-parent conflict is normative and plays an integral part in the adolescent's individuation process (Branje et al. 2013; Grotevant and Cooper 1986). Since adolescents typically expect increased autonomy before parents are ready to grant it (Deković et al. 1997), they might consider conflict as a legitimate means to renegotiate their role in the family (Smetana 1989). Too much conflict is not adaptive, however, as frequent adolescent-parent conflict is correlated with adolescents' antisocial behavior (Klahr et al. 2011b), internalizing and externalizing problems (Branje et al. 2009), and later conduct problems (Klahr et al. 2011a). It is therefore important to identify factors associated with lower levels of conflict.

Adolescent empathy might play a role in this regard. Empathy is a multi-dimensional construct, which encompasses both affective and cognitive dimensions (Davis 1983). Two of these dimensions particularly have been linked to conflict-related constructs. Perspective taking, a cognitive empathy dimension, inhibits aggression in adults (Richardson et al. 1994), and promotes mutually beneficial outcomes in negotiations (Galinsky et al. 2008). Empathic concern, an affective empathy dimension, rouses an urge in adults to reduce others' distress (Stocks et al. 2009). In adolescents, affective empathy has been linked to reduced aggression (De Kemp et al. 2007), as well as greater positive conflict management and reduced escalation in conflicts with peers (De Wied et al. 2007). It remains to be seen whether these findings can be generalized to adolescent-parent relationships. Adolescents' developing empathy might help buffer adolescent-parent conflict, whereas a lack of perspective taking and concern for others might render some teenagers less able to recognize when they are crossing parents' boundaries, resulting in more frequent conflicts.

It is important to acknowledge that the association between adolescent empathy and adolescent-parent conflict might be reciprocal, because the adolescent-parent relationship plays a role in adolescents' empathy development. Adolescents' perceptions of parents' open communication (Heller et al. 2006) and support (Miklikowska et al. 2011) predict adolescents' empathic dispositions, both cross-sectionally and over time. Furthermore, mothers' dispositional perspective taking predicts over-time increases in their daughters' perspective taking (Van Lissa et al. 2014). If supportive, communicative adolescent-parent relationships are positively associated with adolescent empathy development, then frequent adolescent-parent conflict is likely associated with diminished empathy development. The present study investigated the longitudinal association between adolescents' empathy development and the frequency of adolescent-parent conflict. We predicted that relatively greater adolescent empathy would be associated with less adolescent-parent conflict throughout adolescence.

\section{Empathy and Reporter Discrepancies in Perceived Conflict}

Obtaining multiple-informant data is generally considered desirable in family research, but discrepancies between different family members' reports are rarely explained (De Los Reyes and Kazdin 2005). Perspectives on the adolescent-parent relationship may temporarily diverge, particularly in early adolescence (Branje et al. 2013; Steinberg 2001), because adolescents strive towards increasing autonomy (Grotevant and Cooper 1986), while parents are still concerned with instilling proper values in them (Steinberg 2001). Sillars et al. (2010) theory of motivated misunderstanding in family conflicts suggests that such divergent goals can lead parents and adolescents to interpret the same discussions very differently. A metaanalysis by Laursen et al. (1998) provides some empirical support for divergence, as adolescents reported a greater decrease in conflict from early- to late-adolescence than parents. An inspection of dual-reporter longitudinal studies suggests that, following initial agreement in early adolescence, adolescent- and parent reports diverge over time, with adolescents reporting stronger decreases in the frequency of conflict than their parents (Galambos and 
Almeida 1992; Steinberg 1988). In line with these findings, we predicted that that adolescent and parent reports of the frequency of conflict would diverge from early- to mid-adolescence.

Discrepancies in adolescent- and parent-reported conflict may be a risk factor for adolescent adjustment problems, above and beyond those associated with the frequency of conflict. Previous research has shown that mother-adolescent discrepancies on different variables (including indices of relationship quality, parenting behavior, and adolescent problem behavior) predicts adolescent internalizing- and externalizing problems, both concurrently (Ohannessian et al. 2000) and over time (Pelton and Forehand 2001; Pelton et al. 2001; Shek 1998), as well legal, social, and mental health outcomes 4 years later (Ferdinand et al. 2004). These findings highlight the importance of identifying factors associated with larger discrepancies between adolescents' and parents' views of their interactions.

Empathy is likely to play a role in the extent to which different respondents in a family agree about the nature of their interactions. Perspective taking helps individuals understand others' putative states of mind even in the absence of overt signs (Hawk et al. 2011), and might thus help adolescents understand their parents' point of view. Furthermore, affective empathy may enable adolescents to be more responsive to parents' emotional cues that signal anger and disagreement: Modest anger expressions draw attention to the importance of a disagreement, and prompt a focus on finding constructive solutions (Van Kleef et al. 2004). Low affective empathy is associated with reduced sensitivity to such cues, as indicated by decreased mimicry of angry facial expressions, even at the subliminal level (De Wied et al. 2006; Sonnby-Borgström 2002). We thus expected to find a link between adolescent empathy and adolescent-parent discrepancies in reported conflict. Specifically, discrepancies might be larger for lower-empathy adolescents, who more likely fail to consider opposing perspectives and miss emotional cues signaling the importance of a conflict.

\section{Individual Differences in Empathy Development}

There is increasing consensus that adolescence is a developmentally sensitive period for empathy, especially perspective taking (e.g., Van der Graaff et al. 2013; Van Lissa et al. 2014). Neuro-imaging studies suggest that the mechanism underlying this sensitivity may be traced to developmental changes in the prefrontal cortex - an area integral to perspective taking (Choudhury et al. 2006; Singer 2006). However, most studies of empathy development have examined mean-level change, aggregated on the level of an entire sample or separated by sex, and there is little agreement between these studies about the direction of change in adolescence. For example, one study found increases for both empathic concern and perspective taking (Davis and Franzoi 1991), another for perspective taking only (Eisenberg et al. 2005), and one found no change at all (Grühn et al. 2008). Finally, two studies found curvilinear trajectories, with an increase in empathy until age 12 and subsequent decline until age 14 (Lam et al. 2012), and a dip in empathy around age 16 (Van der Graaff et al. 2013), respectively.

Such diversity in findings might suggest that people differ in terms of their trajectories of empathy development. If that is the case, a person-centered analysis might be more appropriate than mean-level analysis, because it can reveal heterogeneity in developmental trajectories (Jung and Wickrama 2008). In line with this explanation, studies using latent growth analysis usually find significant inter-individual variance in the slope of empathy development (e.g., Grühn et al. 2008), which means that participants differ in their rate of change, even if average change for the sample summed to zero. Similarly, crosslagged panel modelling revealed substantial rank-order change in adolescents' dispositional perspective taking (Van Lissa et al. 2014), which means that some adolescents increased more than others. Therefore, it may be useful to identify subgroups, or classes of adolescents with distinct developmental trajectories of empathy, and to compare the frequency of adolescent- and parent-reported conflict between these classes.

\section{The Present Study}

We explored the association between individual differences in empathy development and adolescent- and parent-reported conflict in a 6-year longitudinal study. First, we investigated heterogeneity in empathy development by identifying classes of individuals characterized by similar developmental trajectories. To address the association between empathy and the frequency of conflict, we compared these empathy classes in terms of initial levels and over-time changes in the frequency of adolescent- and parent-reported conflict. We predicted that adolescents with relatively higher empathy would be characterized by less frequent adolescent- and parent-reported conflict over time, compared to adolescents with lower empathy. In order to address the association between empathy and discrepancies between adolescent and parental reports of conflict frequency, we compared the frequency of adolescentand parent-reported conflict within each empathy class. We predicted that there would be temporary reporter divergence between adolescents and both parents from early- to midadolescence. We further expected that this divergence would be greater for adolescents with lower empathy than for those with higher empathy. 
Table 1 Ranges of concurrent correlations between empathy and conflict variables

\begin{tabular}{lllll}
\hline Variable & 1. EC & 2. PT & 3. Conflict AM & 4. Conflict MA \\
\hline 2. Perspective taking & $0.49,0.62$ & & & \\
3. Conflict AM & $-0.12,-0.07$ & $-0.18,-0.07$ & & \\
4. Conflict MA & $-0.18,-0.06$ & $-0.21,-0.05$ & $0.29,0.42$ & $0.33,0.44$ \\
5. Conflict AF & $-0.12,-0.06$ & $-0.17,-0.10$ & $0.62,0.73$ & $0.40,0.48$ \\
6. Conflict FA & $-0.21,-0.10$ & $-0.23,-0.11$ & $0.23,0.34$ & $0.36,0.46$ \\
\hline
\end{tabular}

Empathic concern (EC), perspective taking (PT), adolescent about mother (AM), mother about adolescent (MA), adolescent about father (AF), father about adolescent (FA)

\section{Methods}

Participants

Participants were 467 Dutch adolescents ${ }^{1}$ (266 boys; age at T1: $M=13.03, S D=0.46)$, their mothers $\left(N=467, M_{\text {age }}\right.$ at T1: $M=44.41, S D=4.46)$, and fathers $(N=437$, $\left.M_{\text {age }}=46.74, S D=5.10\right)$, participating in an ongoing longitudinal study (Van Lier et al. 2011). Most adolescents had a Dutch ethnic background $(N=446)$, although some had a Surinamese/Antillean $(N=6)$, or other background ( $N=14 ; 1$ missing). Socio-economic status was based on parents' reports of employment status (Statistics-Netherlands 1993). Ten percent of the families were classified as low-SES, and 90 percent were classified as medium- to high-SES.

\section{Procedure}

The RADAR sample was recruited from randomly selected schools in the province of Utrecht, and four main cities in The Netherlands. Families were randomly selected within these schools, and included in the study if adolescents and parents provided informed consent. Of 1,081 families contacted, 470 refused and 114 failed to produce informed consent. From 2006 to 2012, adolescents and both parents completed yearly questionnaires at home, in the presence of a trained researcher, which included the variables used in the present study. They received financial compensation for their participation at each wave (approximately \$40). At wave six, 422 adolescents (90\% of the sample) were still involved in the study. Average participation over the six waves was $95 \%$.

\footnotetext{
1 Thirty adolescents were omitted from the original sample of 497 , because they had completed the IRI fewer than three times (the minimum required for latent growth analysis) and could thus not be assigned a class membership based on their developmental trajectories. There were no significant differences between the deleted cases and the rest of the sample in terms of adolescent- or parent-reported conflict frequency at any time point (all p's between .09 and .84).
}

Measures

\section{Empathy}

We used two subscales of Davis' (1983) Interpersonal Reactivity Index (IRI) to assess adolescents' empathic concern (EC, "I am often concerned about people less fortunate than me") and perspective taking (PT, "Sometimes I try to understand my friends better by imagining how they see things"). Each subscale contains seven items, rated on a 5-point Likert scale $(0=$ Doesn't describe me at all; 4 = Describes me very well). Previous research found that the Dutch IRI has adequate reliability and external validity in different samples of adults and adolescents (De Corte et al. 2007; Hawk et al. 2012). In the present study, the reliability of empathic concern was acceptable in the first wave $(\alpha=.62)$ and good in all other waves (between .71 and .76). Reliability for perspective taking was good in waves four through six ( $\alpha$ 's between .75 and .78), and acceptable in waves one and two $(\alpha=.60$ and .67). Concurrent correlations between EC and PT ranged between $r=.43$ and .69 (for concurrent correlations with conflict, see Table 1).

Two factors might have contributed to the relatively lower reliability of empathic concern and perspective taking in the first wave. First, Cronbach's alpha becomes lower when scale variance decreases. According to Levene's tests, the scale variance was significantly lower in wave one than in all other waves for both empathic concern, $\mathrm{F}(5,2679)=3.26, p=.01$, and perspective taking, $\mathrm{F}(5,2679)=3.05, p=.01$. Second, empathy is a multidimensional construct with highly correlated subscales. Under such circumstances, Cronbach's alpha can provide a strong underestimation of reliability, because the assumption of tau-equivalence is likely to be violated (Sijtsma 2009). In such cases, the greatest lower bound of reliability may be a better indicator, as it indicates the lowest possible true reliability given the observable covariance matrix. The glb varied between .72 and .83 for empathic concern, and between .71 and .86 for perspective taking, which suggests good reliability at all waves. 


\section{The Frequency of Conflict}

Self-reported conflict frequency was assessed using Laursen's (1993) Interpersonal Conflict Questionnaire (ICQ). Adolescents reported on conflicts with each parent separately, and parents both reported independently on their conflicts with the adolescent. Respondents reported how often conflicts occurred for each of 10 common conflict topics (e.g., "Autonomy, personal freedom", "school/ work", "criticism or teasing") in the past 7 days, on a 5-point Likert scale ( $1=$ Never; $5=$ Often $)$. Cronbach's $\alpha$ of adolescent-reported conflict frequency with parents varied between .84 and .89, and Cronbach's $\alpha$ of parentreported conflict frequency varied between .87 and .92 . For concurrent correlations between reporters, see Table 1.

\section{Results}

\section{Strategy of Analyses}

All analyses were conducted using structural equation modeling in MPlus (Muthén and Muthén 1998-2012). Little's (1988) MCAR test was non-significant, indicating no systematic differences between participants with complete data and participants with partially missing data (between $.8 \%$ at $\mathrm{T} 1$ and $15.5 \%$ at T6), $\chi^{2}(1322)=$ 1358.816, $p=.24$. This indicates that the use of full information maximum likelihood estimation (FIML) was warranted. This procedure makes use of all available information, without estimating missing data. Covariance coverage ranged from .79 to .99 , which is more than enough for reliable model estimation. We considered RMSEA $\leq .05$, and $\mathrm{CFI} \geq .95$, supplemented by SRMR $\leq .08$, to indicate good fit (Kline 2011). The fit of each model is displayed in Table 2. Model fit was compared using $\chi^{2}$-difference tests.

\section{Identifying Developmental Trajectories of Empathy}

To identify classes of adolescents characterized by distinct developmental trajectories of empathic concern and perspective taking, we used Latent Class Growth Analysis (LCGA: Jung and Wickrama 2008). The developmental trajectories of adolescents' empathic concern and perspective taking are represented with latent growth curve models, after which adolescents' class membership is estimated based on the parameters of these latent growth models (i.e., intercept, slope, and quadratic change). Because empathic concern and perspective taking were moderately- to highly correlated in each wave ( $r$ 's between .47 and .62), we included both growth trajectories in a dual-process model, and accounted for shared error variance by correlating the residuals of empathic concern and perspective taking within each wave.

We established that a linear model $\left(\mathrm{M}_{1}\right.$, see Table 2$)$ fit the data worse than a curvilinear model $\left(\mathrm{M}_{2}\right)$. We determined that a three-class model was optimal, by selecting the model with the lowest BIC that still fit better than a model with one class less, based on a significant VLMRtest (see: Jung and Wickrama 2008). Compared to a twoand four-class model, a three-class model also had the

Table 2 Overview of model fit indices

\begin{tabular}{|c|c|c|c|c|c|c|c|c|}
\hline Model & $\chi^{2}$ & $\mathrm{df}$ & BIC & RMSEA & CFI & SRMR & $\Delta \chi^{2}$ & $\mathrm{df}$ \\
\hline \multicolumn{9}{|l|}{ LCGA of empathy } \\
\hline M1: Linear LCGA & 145.01 & 58 & $6,892.42$ & 0.057 & 0.97 & 0.103 & & \\
\hline M2: Quadratic LCGA & 67.33 & 45 & $6,853.38$ & 0.033 & 0.992 & 0.048 & $77.68 * * *$ & 13 \\
\hline M3: Piecewise LGA & 223.023 & 159 & $6,169.32$ & 0.051 & 0.973 & 0.088 & $155.69 * *$ & 114 \\
\hline \multicolumn{9}{|c|}{ LGA of adolescent-mother conflict } \\
\hline M4: Linear LGA & 391.02 & 192 & $8,826.93$ & 0.082 & 0.926 & 0.083 & & \\
\hline M5: Quadratic LGA & 304.98 & 171 & $8,803.31$ & 0.071 & 0.95 & 0.068 & $86.04 * * *$ & 21 \\
\hline M6: Piecewise & 280.98 & 164 & $8,800.12$ & 0.068 & 0.957 & 0.062 & $24.00 * * *$ & 7 \\
\hline \multicolumn{9}{|c|}{ LGA of adolescent-father conflict } \\
\hline M7: Linear LGA & 324.08 & 192 & $7,984.69$ & 0.067 & 0.947 & 0.073 & & \\
\hline M8: Quadratic LGA & 309.85 & 186 & $7,988.20$ & 0.066 & 0.951 & 0.074 & $14.23 *$ & 6 \\
\hline M9: Piecewise & 259.71 & 170 & $7,985.34$ & 0.059 & 0.964 & 0.059 & $50.15 * * *$ & 16 \\
\hline \multicolumn{9}{|c|}{ LGA of mother-father conflict } \\
\hline M10: Linear LGA & 469.40 & 194 & $7,720.19$ & 0.095 & 0.908 & 0.069 & & \\
\hline M11: Quadratic LGA & 448.52 & 195 & $7,696.34$ & 0.091 & 0.915 & 0.079 & $20.88 * * *$ & 1 \\
\hline M12: Piecewise & 393.92 & 176 & $7,698.22$ & 0.089 & 0.927 & 0.061 & $54.60 * * *$ & 19 \\
\hline
\end{tabular}

$* p<.05 ; * * p<.01 ; * * * p<.001$ 
Fig. 1 Developmental trajectories of adolescents' empathic concern (EC) and perspective taking (PT) for the three empathy classes

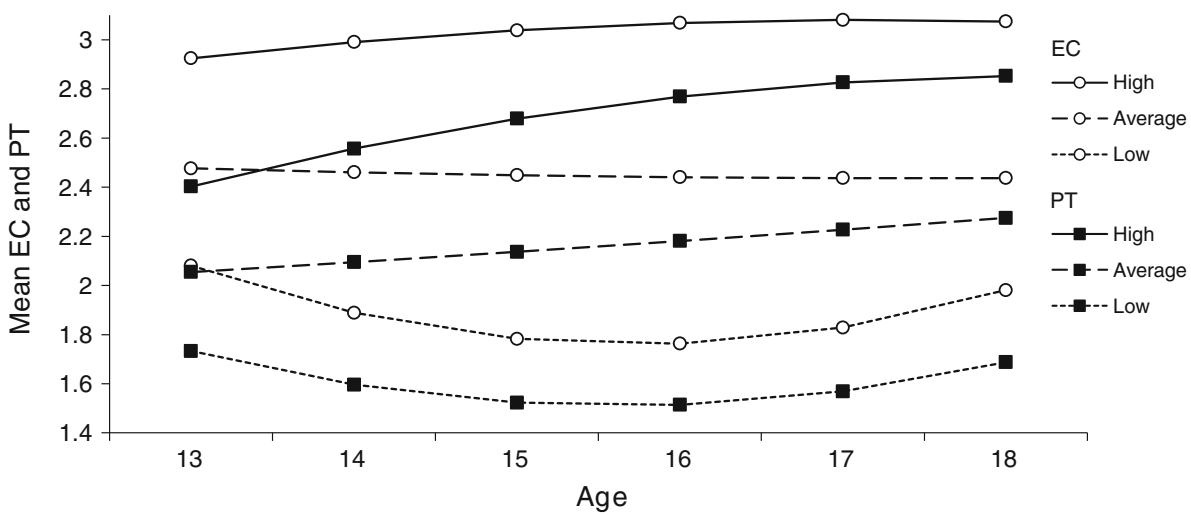

highest entropy $(\alpha=.85)$ and posterior probabilities (between .93 and .94), further indicating good fit. Moreover, the three classes each contained a sizeable number of participants, and their trajectories of empathy development were distinct (Fig. 1). The parameter estimates were replicated twice with different starting values, suggesting that these were not local solutions. To account for known sex differences in empathy development (Van der Graaff et al. 2013), ${ }^{2}$ we included sex (contrast coded) as a predictor of the developmental trajectories and class membership. This approach is similar to conducting the analyses for each sex separately and combining the resulting classes with the corresponding group of the other sex afterwards, with the added benefit that sex differences in developmental trajectories are explicitly modelled and reported. Girls had a significantly higher intercept than boys for empathic concern and perspective taking $(B=0.23$ and $B=0.10$, $p$ 's $<.001)$, a greater slope on both variables $(B=0.09$ and $B=0.11, p$ 's $<.001$ ), and a more negative quadratic term $(B$ 's $=-0.02, p$ 's $<.001)$.

Because curvilinear trajectories are difficult to interpret and compare, we re-analyzed the latent growth part of the LCGA model using piece-wise latent growth analysis (Muthén and Muthén 1998-2012). The curvilinear trajectory is split up into two linear segments, with a shared intercept and two separate slopes. The best fit was obtained when the transition between the two linear trajectories, or "knot", was made at age $16\left(\mathrm{M}_{3}\right)$. Thus, linear change is estimated from age 13 to 16 , which we will refer to as "early-mid adolescence", and from age 16 to 18 , or "midlate adolescence" (the slope loadings are $0,1,2,3,3,3$, and $0,0,0,0,1,2$, respectively).

Next, we interpreted the growth trajectories of the three classes (see Fig. 1 and Table 3). A "high empathy" class $(N=105,29 \%$ girls) was characterized by the highest intercepts for empathic concern and perspective taking, and

\footnotetext{
2 This article examined the influence of sex and pubertal development on developmental trajectories of empathy in the RADAR sample.
}

the highest increase in perspective taking from early- to mid-adolescence. An "average empathy" class $(N=283$, $42 \%$ girls) was characterized by lower, stable empathic concern and a slight increase in perspective taking throughout adolescence. Finally, a "low empathy" class ( $N=79,63 \%$ girls) was characterized by the lowest intercepts for empathic concern and perspective taking, as well as a decrease for both variables from early- to midadolescence, followed by a rebound from mid- to lateadolescence. This u-shaped curve suggests an "empathy dip" around age 16 (Van der Graaff et al. 2013). For empathic concern, only the developmental trajectory of the low-empathy class differed significantly from all other classes throughout adolescence, $\chi_{\text {Wald }}^{2}$ 's between 14.41 and 23.10, $p$ 's $<.001$. The trajectories of the average- and high-empathy group did not differ significantly from one another, $\chi_{\text {Wald }}^{2}$ 's(1) between 0.001 and 2.87 , $p$ 's between .09 and .98 . This suggests that low-empathy adolescents experience a temporary dip in empathic concern. For perspective taking, the trajectories of all classes differed significantly from early- to mid-adolescence, $\chi_{\text {Wald }}^{2}$ 's between 8.65 and $35.56, p$ 's $<.003$. High-empathy adolescents increased the most, followed by average-empathy adolescents, while low-empathy adolescents decreased in perspective taking. From mid- to late-adolescence, there were no significant differences between classes, $\chi_{\text {Wald }}^{2}$ 's between $=0.001$ and 2.04, $p$ 's between .15 and .97 . This suggests that from early- to mid-adolescence, the classes became further differentiated in terms of PT, and that these amplified differences subsequently remained stable. The distribution of sex across classes was unequal, $\chi^{2}(2)=22.19, p<.001$. After taking into account sex differences in empathy development, girls were relatively overrepresented in the low-empathy class. This suggests that, even though girls on average have greater empathy than boys, the distribution of empathy within gender was skewed. This means that, in comparison to girls whose empathy scores were around girls' average levels, there were relatively more girls with lower empathy. 
Table 3 Latent variable means by empathy class

\begin{tabular}{|c|c|c|c|c|c|c|c|c|c|c|c|}
\hline \multirow[t]{2}{*}{ Empathy class } & \multirow[t]{2}{*}{ Variable } & \multicolumn{2}{|c|}{ Adolescent empathy } & \multicolumn{4}{|c|}{ Adolescent-mother conflict } & \multicolumn{4}{|c|}{ Adolescent-father conflict } \\
\hline & & $\mathrm{EC}$ & PT & Adolescent & Mother & $\Delta \chi^{2}$ & $p$ & Adolescent & Father & $\Delta \chi^{2}$ & $p$ \\
\hline \multirow[t]{3}{*}{ Low } & Intercept & $2.06 * * *$ & $1.71 * * *$ & $2.43 * * *$ & $2.50 * * *$ & 0.56 & 0.45 & $2.39 * * *$ & $2.37 * * *$ & 0.08 & 0.79 \\
\hline & Slope 1 & $-0.13^{* * *}$ & $-0.08 * * *$ & $-0.07^{*}$ & 0.03 & 5.51 & 0.02 & -0.05 & 0.02 & 4.34 & 0.04 \\
\hline & Slope 2 & $0.16^{* * *}$ & $0.11 * *$ & -0.03 & $-0.13^{* * *}$ & 3.13 & 0.08 & $-0.08 *$ & $-0.14 * * *$ & 1.35 & 0.25 \\
\hline \multirow[t]{3}{*}{ Average } & Intercept & $2.48 * * *$ & $2.05 * * *$ & $2.17 * * *$ & $2.20 * * *$ & 0.53 & 0.47 & $2.15^{* * *}$ & $2.14 * * *$ & 0.09 & 0.76 \\
\hline & Slope 1 & -0.02 & $0.04 * * *$ & $-0.04 *$ & 0.01 & 5.78 & 0.02 & $-0.04 * *$ & -0.01 & 3.46 & 0.06 \\
\hline & Slope 2 & 0.01 & $0.05^{* *}$ & $-0.05^{*}$ & $-0.10^{* * *}$ & 3.13 & 0.08 & $-0.07 * * *$ & $-0.05^{*}$ & 0.57 & 0.45 \\
\hline \multirow[t]{3}{*}{ High } & Intercept & $2.99 * * *$ & $2.46 * * *$ & $2.08 * * *$ & $2.12 * * *$ & 0.27 & 0.6 & $2.03 * * *$ & $2.08 * * *$ & 0.51 & 0.47 \\
\hline & Slope 1 & 0.03 & $0.12 * * *$ & -0.03 & 0.02 & 2.45 & 0.12 & -0.04 & -0.02 & 0.63 & 0.43 \\
\hline & Slope 2 & 0.01 & 0.05 & 0 & $-0.08 * *$ & 4.08 & 0.04 & 0 & -0.03 & 0.62 & 0.43 \\
\hline
\end{tabular}

$* p<.05 ; * * p<.01 ; * * * p<.001$

Differences in Adolescent-Parent Conflict between Empathy Classes

We hypothesized that the frequency of adolescent-parent conflict would differ between the different empathy classes, and that there would be greater divergence between the frequency of adolescent- and parent-reported conflict in lower-empathy classes than in higher-empathy classes. We conducted multi-group latent growth analyses of adolescent- and parent-reported conflict frequency. To account for the dyadic nature of the data, we again used a dualprocess model, and correlated the latent growth factors of both respondents. Because of limited sample size, separate analyses were conducted to compare adolescent- and mother reports, adolescent- and father reports, and motherand father reports. In all cases, curvilinear models $\left(\mathrm{M}_{5}, \mathrm{M}_{8}\right.$, $\left.\mathrm{M}_{11}\right)$ fit the data better than linear models $\left(\mathrm{M}_{4}, \mathrm{M}_{7}, \mathrm{M}_{10}\right)$. For the sake of interpretability, we re-analyzed the data using piece-wise latent growth analysis. In all cases, the best fit was obtained with linear trajectories from age 13 to 16 and 16 to $18\left(\mathrm{M}_{6}, \mathrm{M}_{9}, \mathrm{M}_{12}\right)$. In order to test our hypotheses, we then used Wald tests to compare the means of the growth curves' intercepts and slopes between adolescents and parents within each empathy class, and within adolescents and parents, respectively, between the empathy classes.

\section{The Frequency of Conflict}

We tested the hypothesis that adolescents with lower empathy and both of their parents would report greater conflict frequency than adolescents with higher empathy and their parents.

Adolescents and Mothers In line with our hypothesis, we found that the intercepts of conflict frequency were significantly higher for low-empathy adolescents and their mothers than for all others, all $\chi_{\text {Wald }}^{2}(1) \geq 8.53$, all $p$ 's $\leq .004$. There were no significant differences in the intercepts of conflict frequency between average- and highempathy adolescents, nor between the mothers in these groups, all $\chi_{\text {Wald }}^{2}(1) \geq 1.39$, all $p$ 's $\geq .24$. This suggests that, at age 13, low-empathy adolescents and their mothers reported significantly more frequent conflict than all others. For both adolescents and mothers, the slopes of conflict frequency did not differ significantly between the empathy classes, neither from early- to mid-adolescence ( $p$ 's between .37 and .76), nor from mid-to late-adolescence ( $p$ 's between .19 and .67), indicating that these initial differences persisted over time.

Adolescents and Fathers As with mothers, we found that the intercepts of conflict frequency were significantly higher for low-empathy adolescents and their fathers than for all others, all $\chi_{\text {Wald }}^{2}(1) \geq 6.86$, all $p$ 's $<.01$. There were no significant differences between the intercepts of conflict frequency between average- and high-empathy adolescents $(p=.11)$, nor between the fathers in these groups $(p=.38)$. This suggests that, at age 13 , low-empathy adolescents and their fathers reported significantly more frequent conflict than all other groups. From early- to midadolescence, the slopes of conflict frequency did not differ between the empathy classes for both adolescents and fathers ( $p$ 's between .26 and .86). From mid-to late-adolescence, only the slopes of average- and high-empathy adolescents differed significantly, $\quad \chi_{\text {Wald }}^{2}(1)=3.944$, $p=.047$. Average-empathy adolescents displayed a significant decrease in conflict, whereas high-empathy adolescents' conflict remained stable. For fathers, only the slopes of fathers in the low-empathy class differed significantly from those in the high- and average-empathy classes, $\quad \chi_{\text {Wald }}^{2}(1)=4.37, \quad p=.04 \quad$ and $\chi_{\text {Wald }}^{2}(1)=3.70$, $p=.05$, respectively. Low-empathy fathers reported a 


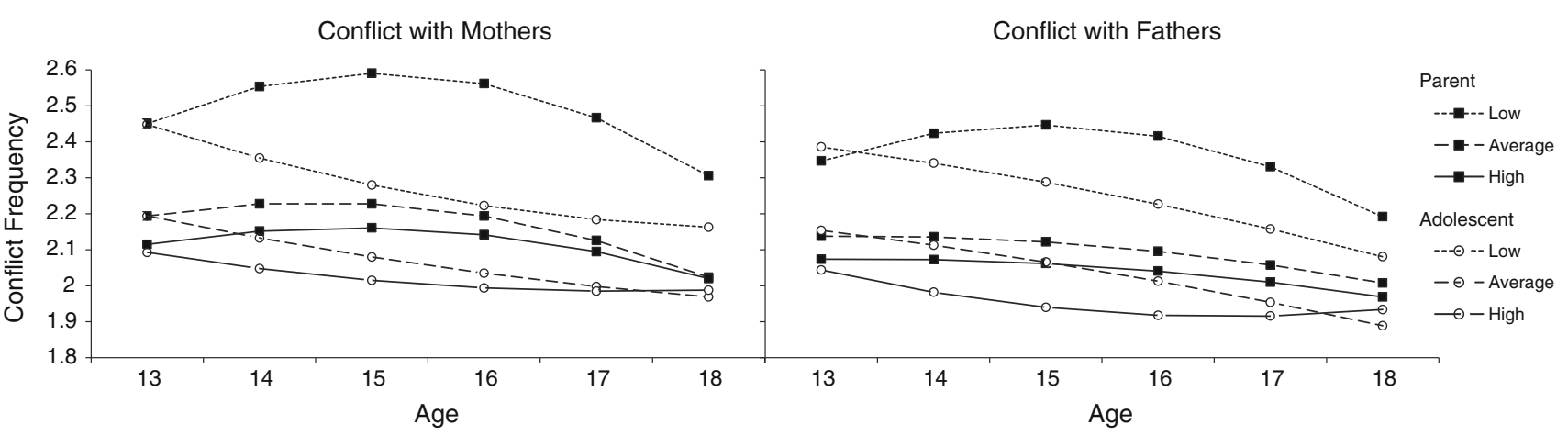

Fig. 2 The frequency of conflict reported by adolescents and their parents, grouped by empathy class

significant decrease in conflict, whereas high-empathy fathers remained stable, and average-empathy fathers reported a smaller decline. This suggests that, from mid- to late-adolescence, low-empathy fathers' reported conflict decreased more than other fathers' conflict.

\section{Reporter Discrepancies}

We tested the hypothesis that there would be temporary divergence between the reports of adolescents and both parents, and that this divergence would be greater for adolescents with lower empathy than for those with higher empathy.

Adolescents and Mothers There were no significant differences between the intercepts of mother- and adolescentreported conflict in any of the classes (all $p$ 's $>.45$ ), which suggests that mothers and adolescents in all classes were in agreement about the frequency of conflict at age 13. From early- to mid-adolescence, however, in both the low- and the average-empathy classes, the slopes of conflict frequency of adolescents and their mothers significantly differed, $\chi_{\text {Wald }}^{2}(1)=5.51, \quad p=.02$ and $\chi_{\text {Wald }}^{2}(1)=5.78$, $p=.02$, respectively. Specifically, low- and averageempathy adolescents reported a significant decrease in conflict, while their mothers reported stability (see Table 3). This indicates that, from early- to mid-adolescence, adolescent- and mother-reported conflict diverged for low- and average-empathy adolescents. Slopes did not differ significantly for high-empathy adolescents and their mothers, $\chi_{\text {Wald }}^{2}(1)=2.45, p=.12$.

From mid- to late-adolescence, the difference between the slopes of adolescent- and mother-reported conflict bordered on significance for low- and average-empathy adolescents, both $\chi_{\mathrm{Wald}}^{2}(1)=3.13, p$ 's $=.08$. Low-empathy adolescents reported stability, whereas their mothers reported a decrease. Average-empathy adolescents and their mothers both reported a decrease, which was stronger for mothers than for adolescents. These results suggest a trend towards convergence in both classes. Finally, there was a significant difference in slopes for high-empathy adolescents and their mothers, $\chi_{\text {Wald }}^{2}(1)=4.08, p=.04$, with adolescents reporting stability whereas their mothers reported a significant decrease. Although their reports of conflict were relatively stable from early- to mid-adolescence, their slopes were opposite in valence, leading them to drift apart slightly, but not significantly (see Fig. 2). The significant difference in slopes from mid- to late-adolescence can be attributed to the sharp re-convergence of their reports, which occurred within a shorter period. These findings are in line with our hypothesis that adolescent- and parent-reported conflict would temporarily diverge, and that this divergence would be greater for adolescents with lower empathy than for those with higher empathy. Specifically, we found evidence of such divergence for low- and averageempathy adolescents, but not for high-empathy adolescents.

Adolescents and Fathers There were no differences between the intercepts of father- and adolescent-reported conflict frequency in any of the classes (all $p$ 's > .47), indicating adolescent-father agreement about the frequency of conflict at age 13. From early- to mid-adolescence, however, the slopes of conflict frequency significantly differed for low-empathy adolescents and their fathers, $\chi_{\text {Wald }}^{2}(1)=4.34, p=.04$. Although the slopes of lowempathy adolescents and their fathers were non-significant, they were opposite in valence, with a negative slope for adolescents, and a positive slope for fathers. For averageempathy adolescents and their fathers, the difference was only borderline significant, $\chi_{\text {Wald }}^{2}(1)=3.46, p=.06$. Average-empathy adolescents demonstrated a small, significant decrease in conflict from early- to mid-adolescence, while their fathers reported stability. These results suggest that adolescent-father perspectives on conflict diverged slightly from early- to mid-adolescence for the low- and average-empathy classes. High-empathy adolescents and their fathers' slopes did not differ significantly, $\chi_{\text {Wald }}^{2}(1)=0.63, p=.43$, and their slopes were not 
significant, indicating that their reports of conflict were relatively stable in this period. This suggests that highempathy adolescents and their fathers were in agreement about the frequency and (lack of) change in conflict from early- to mid-adolescence.

From mid- to late-adolescence, there were no significant differences between the slopes of adolescent- and fatherreported conflict in any of the empathy classes, all $\chi_{\text {Wald }}^{2}(1) \leq 1.35, \quad p$ 's $\geq .25$. Low- and average-empathy adolescents and their fathers all reported a significant decrease in conflict, which suggests that they were in agreement about the diminishing frequency of conflict. High-empathy adolescents and their fathers both reported stability. These findings offer partial support for our hypothesis that adolescent- and parent reports of the frequency of conflict would temporarily diverge, and that this divergence would be greater for adolescents with lower empathy than for those with higher empathy. Specifically, we found evidence for reporter divergence in the low- and average-empathy classes, but not the high-empathy class. Adolescent- and father-reported conflict did not re-converge from mid-to late-adolescence. Instead, adolescents and fathers were in agreement about the (lack of) change during this period.

Mothers and Fathers There were no differences between mother- and father-reported conflict frequency within any of the classes in terms of the intercepts $(.11 \leq p$ 's $\leq .71)$, slopes from early- to mid-adolescence $(.20 \leq p$ 's $\leq .93)$, or slopes from mid- to late-adolescence $(.06 \leq p$ 's $\leq .75)$. This suggests that parents within each empathy class were in agreement with one another about the frequency and change in conflict throughout adolescence.

\section{Discussion}

The present study set out to examine the relationship between adolescents' developing empathy and the frequency of conflict with parents, as well as the level of agreement between adolescents and parents about the frequency of such conflicts. While some adolescent-parent conflict is normative, frequent conflict is associated with adolescent maladjustment (Branje et al. 2009). Empathy has been linked with conflict-related constructs in adultand adolescent peer relationships (De Wied et al. 2007; Richardson et al. 1994), but the present study is the first to address this association in the adolescent-parent relationship. Because adolescent and parental reports on their relationship often differ (De Los Reyes and Kazdin 2005), it is important to obtain both adolescent and parent reports of the frequency of conflict. Discrepancies between their reports might be explained in part by adolescents' developing empathy.
Our findings suggest that, particularly in early adolescence, individual differences in empathic dispositions become further amplified, and that these differences in empathic dispositions are associated with both the frequency of adolescent-parent conflict, and adolescent-parent agreement about the frequency of conflict. In particular, low-empathy adolescents and their parents reported significantly more conflict than all others throughout adolescence. Regarding reporter discrepancies, we found that mothers' and fathers' reports of the frequency of conflict were in agreement throughout adolescence in all empathy classes. High-empathy adolescents' reports were also in line with those of their parents. Low- and average-empathy adolescents' reports, however, diverged temporarily from their parents' from early- to mid-adolescence, with adolescents reporting decreasing conflict, while both parents agreed that conflict was higher and stable. The finding that low- and average-empathy adolescents' reports diverged from their parents' reports, but that their parents were in agreement with one another, may suggest that these discrepancies are related to distortions in adolescents' perceptions, as opposed to biased parental reports. Interestingly, our results showed that only low-empathy adolescents experienced elevated conflict, whereas only high-empathy adolescents were in agreement with parents about the frequency of conflict throughout adolescence. Average-empathy adolescents were similar to high-empathy adolescents in the sense that they did not show elevated conflict, and similar to low-empathy adolescents in the sense that their reports diverged from parents' over time. This suggests that only low empathy is associated with increased adolescent-parent conflict, while high empathy is required for adolescent-parent agreement.

\section{Heterogeneity of Empathy Development}

Whereas previous research on empathy development has typically examined mean-level change, the present study took a person-centered approach to identify classes of adolescents with similar developmental trajectories. We identified a "high-empathy" class, with high, stable empathic concern and high-increasing perspective taking, an "average-empathy" class with stable empathic concern and slightly increasing perspective taking, and a "lowempathy" class, with a dip in both variables around midadolescence. The differences between these trajectories suggest that initial differences in perspective taking become further amplified from early- to mid-adolescence. This finding builds on previous research that suggests adolescence is a developmentally sensitive period for perspective taking (e.g., Choudhury et al. 2006; Van Lissa et al. 2014) by indicating that development is not uniform for all adolescents, but that further differentiation between 
individuals occurs. This pattern is in line with the process of "accentuation" proposed by Block (1982), by which individual differences are accentuated during times of transition. It might further be explained in the context of Choudhury et al. (2006) suggestion that social experiences in adolescence interact with changes in the brain to hone the development of perspective taking strategies. These results suggest that early empathy levels may be a marker for their later developmental trajectory. For clinicians, this highlights the potential importance of detecting lower empathy at an early age, and providing interventions that support empathy development to prevent low-empathy adolescents from falling further behind their peers over time.

Our finding that girls were overrepresented in the lowempathy class may at first glance appear counterintuitive, in light of the well-established finding that girls often report being more empathic (Davis 1983). However, we specifically controlled for sex differences in our analyses, to prevent class membership from being based primarily on known sex differences in developmental trajectories of empathy (Jung and Wickrama 2008). Our low-empathy class therefore consists of girls whose empathy was low compared to other girls, and boys whose empathy was low relative to other boys. The overrepresentation of girls in the low-empathy class suggests that the distribution of empathy within each sex is slightly skewed: There may be relatively many boys with high empathy compared boys' average empathy level, and relatively many girls with low empathy compared to girls' average empathy level.

\section{Empathy and the Frequency of Adolescent-Parent Conflict}

The present study provides the first evidence for a link between adolescent empathy development, and the frequency of adolescent-parent conflict. Although previous research has linked empathy to different conflict-related constructs, such as reduced aggression and greater constructive conflict resolution behavior, in the context of adolescent peer- and adult relationships (De Wied et al. 2007; Richardson et al. 1994). The present research expands on this previous work by assessing the frequency of conflict directly, and demonstrating that the link between empathy and conflict can be generalized to the adolescent-parent context. These findings may be relevant for childcare officials and clinicians, because it suggests that lower adolescent empathy may be reflective of a problematic relationship with parents, or conversely, that promoting adolescents' empathy development may help them address conflict with parents more effectively.
Empathy and Discrepancies Between Adolescentand Parent-Reported Conflict

The present study builds upon earlier theoretical and empirical work about discrepancies between adolescentand parent-reported conflict (De Los Reyes and Kazdin 2005; Ehrlich et al. 2011), by suggesting that adolescents' empathic abilities may play a role in explaining such discrepancies. Although discrepancies between adolescentand parent-reported conflict emerged in the low- and average-empathy classes, high-empathy adolescents' reports were mostly in agreement with their parents' throughout adolescence. A relatively steeper increase in perspective taking set the high-empathy class apart from the low- and average-empathy classes. Perhaps this steeper increase in perspective taking allowed high-empathy adolescents to continue considering their parents' concerns, and maintain closer bonds while they navigate the road toward increased independence (Grotevant and Cooper 1986). Low- and average-empathy adolescents, on the other hand, might focus more on their own autonomy gains when parents "give in" in conflicts, and afterwards no longer see the interaction as a conflict. Failure to consider their parents' different perspectives and negative emotions might render them relatively "conflict blind". Perspective taking increased more gradually in average-empathy adolescents than in high-empathy adolescents, and only increased from mid- to late- adolescence in low-empathy adolescents, which may explain the later re-convergence (for mothers) or agreement about change (for fathers) in adolescent- and parent-reported conflict for these empathy classes.

Although our finding that parents reported greater conflict than adolescents is in line with previous dual-reporter longitudinal studies (Galambos and Almeida 1992; Steinberg 1988), at least three notable studies have instead found that adolescents reported more conflict than parents (Molina and Chassin 1996; Smetana 1989). This illustrates that reporter discrepancies remain a complex matter that can stem from many factors. Ethnicity, for example, appears to affect the direction of reporter discrepancies (Walton et al. 1999), because cultures differ in the extent to which they perceive certain behaviors as problematic. Specifically, African-, Hispanic-, and Asian-American adolescents are known to perceive their parents as more authoritarian than Caucasian adolescents (Dornbusch et al. 1987), which might lead them to report greater adolescentparent conflict. Molina and Chassin's (1996) sample contained a large amount of Hispanic families, and the sample of Gonzales et al. (1996) consisted entirely of AfricanAmerican mother-adolescent dyads, which might explain why they found greater adolescent- than parent-reported conflict. Our sample, in contrast, consisted largely of native 
Dutch participants, who may be less authoritarian than even Caucasian-American parents (e.g., Pels and Nijsten 2003). Another factor shared by all three studies finding greater adolescent-reported conflict is that the research was conducted in the presence of an interviewer (Molina and Chassin 1996; Smetana 1989) or a video camera (Gonzales et al. 1996). Family conflict is a delicate matter that is likely to be subject to social desirability bias, and parents especially may underplay conflicts if they are concerned with how their family comes across to researchers. This may especially play a role when parent participants originate from stigmatized populations, such as the alcoholic parents in Molina and Chassin's (1996) study. In our own research, adolescents and parents completed a questionnaire anonymously, which is likely to reduce the influence of social desirability.

The issue of reporter discrepancies is of prime importance in the clinical setting, and in fact most of the relevant literature has taken a clinical approach (see: De Los Reyes and Kazdin 2005). De Los Reyes and Kadzin have argued that adolescent-parent agreement should be greater regarding externalizing- than internalizing problems, because externalizing symptoms are more readily observable. The present study nevertheless found significant discrepancies between conflict reported by low- and average-empathy adolescents and their parents. This suggests that reports can differ, even when it comes to readily observable behaviors. The fact that parents were always in agreement about the frequency of conflict, whereas only high-empathy adolescents were in agreement with their parents, may suggest that lower-empathy adolescents construe conflicts differently, or fail to notice when they cross their parents' boundaries. This highlights the importance of obtaining multiple informants' reports on adolescent behavior in the clinical setting.

\section{Strengths and Limitations}

One strength of the present study is that it included longitudinal data from multiple family members, spanning the entire period from early- to late-adolescence. This is important, because developmental changes in adolescence are known to occur for both empathy (Choudhury et al. 2006) and adolescent-parent conflict (Laursen et al. 1998). Independent assessments of conflict between adolescents and each parent provide a more complete understanding of the frequency of family conflict. Furthermore, although many researchers value the use of multiple-respondent data, these data are often aggregated without considering potentially meaningful discrepancies between them (De Los Reyes and Kazdin 2005). We used dual-trajectory modeling for adolescent- and parent-reported conflict, which allowed us to study trajectories of conflict frequency and reporter discrepancies in a single model, while avoiding the problems associated with difference scores. Finally, De Los Reyes and Kazdin (2005) argued that reporter discrepancies are likely to be greater for less observable behaviors (like internalizing problems) than for highly observable behaviors (like externalizing problems). By that logic, an important strength of the present study is that we demonstrated that adolescent empathy was associated with reporter discrepancies in adolescent-parent conflict, even though conflict is a highly observable behavior, which means that reporter discrepancies are likely to be small. Future research should therefore address whether empathy is related to discrepancies in other, less observable aspects of the adolescent-parent relationship, and whether these findings can be generalized to other close relationships.

One limitation of the present research is that it cannot speak to causality in the link between empathy and conflict. Future research might assess the directionality of this association, for example by investigating whether promoting adolescent empathy helps reduce adolescent-parent conflict, or whether reducing adolescent-parent conflict promotes empathy development. Second, it would have been interesting to investigate the interaction between adolescents' and parents' empathy levels, and to assess how different combinations of adolescent- and parent empathy are associated with perceptions of conflict. However, we were prevented from doing so by a lack of parental empathy measurements, and our limited sample size. Associations between adolescents' and parents' empathy are typically modest (correlations around .15; Hawk et al. 2012; Soenens et al. 2012; Van Lissa et al. 2014), which suggests that all different combinations of adolescent- and parent empathy might exist. To address this matter, future research would therefore require a far larger sample in order to investigate the interaction between parental empathy and adolescent empathy. Another potential limitation was that, according to Cronbach's alpha, the reliability of empathic concern and perspective taking was relatively low at wave one. This might be related to the lower scale variance in this wave, as Cronbach's alpha is contingent on scale variance. Indeed, according to the latent class analysis, the classes were most similar in the first wave (see Fig. 1). Although the glb suggested that reliability might be good, future research should include a measure of empathic dispositions that is more sensitive to individual differences at younger ages, to ensure adequate reliability. Finally, we did not obtain independent observer reports on adolescent-parent conflict, to serve as a more objective measure of the frequency of conflict. This may have indicated whether the reporter discrepancies observed in the low- and average-empathy classes were a result of adolescents underreporting, or 
parents over-reporting conflict. However, the finding that parents were always in agreement with one another, whereas adolescents' agreement with parents was moderated by empathy class, seems to suggest that lowerempathy may be relatively "conflict blind".

\section{Conclusion}

Our findings suggest that initial empathy differences become amplified throughout adolescence. Those who started out with higher empathy increased the most, those who started average remained relatively stable, and those who started low even decreased temporarily, and never reached the levels shown by other groups. Adolescent-parent conflict was more frequent in the low-empathy class than in the highand average-empathy classes. Temporary reporter discrepancies emerged in the low- and average-empathy classes, as adolescents reported decreasing conflict, while their parents reported an increase or stability. These findings extend our understanding of the link between empathy and conflict to the context of adolescent-parent relationships. Furthermore, these results suggest that empathy may play a role in aligning adolescents' and parents' views on their relationship during a time when their goals diverge. These findings suggest the importance of detecting potential empathy deficiencies in childhood, and promoting empathy development before individual differences become amplified. Moreover, low empathy may be an indicator of elevated family conflict, and conversely, promoting adolescents' empathy may help them address conflict with parents more effectively. Finally, this study highlights the importance of obtaining multiple informant reports-especially when "the kids aren't all right". Even when it comes to aspects of the adolescentparent relationship that appear to be highly observable, such as conflict, lower empathy may lead adolescents to fail to see their parents' side of things.

Acknowledgments Data of the RADAR study were used. RADAR has been financially supported by main grants from the Netherlands Organisation for Scientific Research (GB-MAGW 480-03-005, GBMAGW 480-08-006), and Stichting Achmea Slachtoffer en Samenleving (SASS), and various other grants from the Netherlands Organisation for Scientific Research, the VU University Amsterdam and Utrecht University.

Author contributions CJ conceived of the study, conducted the analyses and drafted the manuscript; $\mathrm{SH}$ wrote the grant proposal and supervised the analyses and manuscript writing; SB participated in the design of the longitudinal study and gave feedback on each version of the manuscript; HK participated in the design of the longitudinal study; PL participated in the design of the longitudinal study and gave feedback on the manuscript; WM participated in the design of the longitudinal study and supervised the analyses and manuscript writing. All authors read and approved the final manuscript.

\section{References}

Block, J. (1982). Assimilation, accommodation, and the dynamics of personality development. Child Development, 53, 281-295.

Branje, S. J. T., Laursen, B., \& Collins, W. A. (2013). Parent-child communication during adolescence. In A. L. Vangelisti (Ed.), (2nd ed., pp. 271-286). New York, NY: Routledge.

Branje, S. J. T., van Doorn, M., van der Valk, I., \& Meeus, W. (2009). Parent-adolescent conflicts, conflict resolution types, and adolescent adjustment. Journal of Applied Developmental Psychology, 30(2), 195-204. doi:10.1016/j.appdev.2008.12.004.

Caspi, A., \& Moffitt, T. E. (1991). Individual differences are accentuated during periods of social change: The sample case of girls at puberty. Journal of Personality and Social Psychology, 61(1), 157.

Choudhury, S., Blakemore, S., \& Charman, T. (2006). Social cognitive development during adolescence. Social Cognitive and Affective Neuroscience, 1(3), 165-174. doi:10.1093/scan/ ns1024.

Davis, M. H. (1983). Measuring individual differences in empathy: Evidence for a multidimensional approach. Journal of Personality and Social Psychology, 44(1), 113-126. doi:10.1037/00223514.44.1.113.

Davis, M. H., \& Franzoi, S. L. (1991). Stability and change in adolescent self-consciousness and empathy. Journal of Research in Personality, 25(1), 70-87. doi:10.1016/0092-6566(91)90006-C.

De Corte, K., Buysse, A., Verhofstadt, L., Roeyers, H., Ponnet, K., \& Davis, M. (2007). Measuring empathic tendencies: Reliability and validity of the Dutch version of the interpersonal reactivity index. Psychologica Belgica, 47(4), 235-260.

De Kemp, R. A. T., Overbeek, G., De Wied, M., Engels, R. C. M. E., \& Scholte, R. H. J. (2007). Early adolescent empathy, parental support, and antisocial behavior. The Journal of Genetic Psychology, 168(1), 5-18. doi:10.3200/GNTP.168.1.5-18.

De Los Reyes, A., \& Kazdin, A. E. (2005). Informant discrepancies in the assessment of childhood psychopathology: A critical review, theoretical framework, and recommendations for further study. Psychological Bulletin, 103(4), 483-509. doi:10.1037/00332909.131.4.483.

De Wied, M., Branje, S. J. T., \& Meeus, W. (2007). Empathy and conflict resolution in friendship relations among adolescents. Aggressive Behavior, 33(1), 48-55. doi:10.1002/ab.20166.

De Wied, M., Van Boxtel, A., Zaalberg, R., Goudena, P. P., \& Matthys, W. (2006). Facial EMG responses to dynamic emotional facial expressions in boys with disruptive behavior disorders. Journal of Psychiatric Research, 40(2), 112-121. doi:10.1016/j.jpsychires.2005.08.003.

Deković, M., Noom, M. J., \& Meeus, W. (1997). Expectations regarding development during adolescence: Parental and adolescent perceptions. Journal of Youth and Adolescence, 26(3), 253-272.

Dornbusch, S. M., Ritter, P. L., Leiderman, P. H., Roberts, D. F., \& Fraleigh, M. J. (1987). The relation of parenting style to adolescent school performance. Child Development, 58(5), 1244-1257. doi:10.2307/1130618.

Ehrlich, K. B., Cassidy, J., \& Dykas, M. J. (2011). Reporter discrepancies among parents, adolescents, and peers: Adolescent attachment and informant depressive symptoms as explanatory factors. Child Development, 82(3), 999-1012. doi:10.1111/j. 1467-8624.2010.01530.x.

Eisenberg, N., Cumberland, A., Guthrie, I. K., Murphy, B. C., \& Shepard, S. A. (2005). Age changes in prosocial responding and moral reasoning in adolescence and early adulthood. Journal of Research on Adolescence, 15(3), 235-260. doi:10.1111/j.15327795.2005.00095.x. 
Ferdinand, R. F., Van der Ende, J., \& Verhulst, F. C. (2004). Parentadolescent disagreement regarding psychopathology in adolescents from the general population as a risk factor for adverse outcome. Journal of Abnormal Psychology, 113(2), 198-206.

Galambos, N. L., \& Almeida, D. M. (1992). Does parent-adolescent conflict increase in early adolescence? Journal of Marriage and Family, 54(4), 737-747. http://www.jstor.org/stable/353157.

Galinsky, A. D., Maddux, W. W., Gilin, D., \& White, J. B. (2008). Why it pays to get inside the head of your opponent. Psychological Science, 19(4), 378-384. doi:10.1111/j.14679280.2008.02096.x.

Gonzales, N. A., Cauce, A. M., \& Mason, C. A. (1996). Interobserver agreement in the assessment of parental behavior and parentadolescent conflict: African American mothers, daughters, and independent observers. Child Development, 67(4), 1483-1498. doi: $10.2307 / 1131713$.

Grotevant, H. D., \& Cooper, C. R. (1986). Individuation in family relationships: A perspective on individual differences in the development of identity and role-taking skill in adolescence. Human Development, 29(2), 82-100. doi:10.1159/000273025.

Grühn, D., Rebucal, K., Diehl, M., Lumley, M., \& Labouvie-Vief, G. (2008). Empathy across the adult lifespan: Longitudinal and experience-sampling findings. Emotion, 8(6), 753-765. doi:10. 1037/a0014123.

Hawk, S. T., Fischer, A. H., \& Van Kleef, G. A. (2011). Taking your place or matching your face: Two paths to empathic embarrassment. Emotion, 11(3), 502-513. doi:10.1037/a0022762.

Hawk, S. T., Keijsers, L., Branje, S. J. T., Van der Graaff, J., De Wied, M., \& Meeus, W. (2012). Examining the interpersonal reactivity index (IRI) among early and late adolescents and their mothers. Journal of Personality Assessment, 95, 96-106. doi:10. 1080/00223891.2012.696080.

Heller, S. R., Robinson, L. C., Henry, C. S., \& Plunkett, S. W. (2006). Gender differences in adolescent perceptions of parent-adolescent openness in communication and adolescent empathy. Marriage \& Family Review, 40(4), 103-122. doi:10.1300/J002v40n04.

Jung, T., \& Wickrama, K. A. S. (2008). An introduction to latent class growth analysis and growth mixture modeling. Social and Personality Psychology Compass, 2(1), 302-317. doi:10.1111/j. 1751-9004.2007.00054.x.

Klahr, A. M., McGue, M., Iacono, W. G., \& Burt, S. A. (2011a). The association between parent-child conflict and adolescent conduct problems over time: Results from a longitudinal adoption study. Journal of Abnormal Psychology, 120(1), 46-56. doi:10.1037/ a0021350.

Klahr, A. M., Rueter, M. A., McGue, M., Iacono, W. G., \& Burt, S. A. (2011b). The relationship between parent-child conflict and adolescent antisocial behavior: Confirming shared environmental mediation. Journal of Abnormal Child Psychology, 39(5), 683-694. doi:10.1007/s10802-011-9505-7.

Kline, R. B. (2011). Principles and practice of structural equation modeling. New York: Guilford Press.

Lam, C. B., Solmeyer, A. R., \& McHale, S. M. (2012). Sibling relationships and empathy across the transition to adolescence. Journal of Youth and Adolescence, 41(12), 1657-1670. doi:10. 1007/s10964-012-9781-8.

Laursen, B. (1993). The perceived impact of conflict on adolescent relationships. Merrill-Palmer Quarterly, 39(4), 535-550.

Laursen, B., Coy, K. C., \& Collins, W. A. (1998). Reconsidering changes in parent-child conflict across adolescence: A metaanalysis. Child Development, 69(3), 817-832. doi:10.1111/j. 1467-8624.1998.tb06245.x.

Little, R. J. A. (1988). A test of missing completely at random for multivariate data with missing values. Journal of the American Statistical Association, 83(404), 1198-1202. doi:10.2307/ 2290157.
Miklikowska, M., Duriez, B., \& Soenens, B. (2011). Family roots of empathy-related characteristics: The role of perceived maternal and paternal need support in adolescence. Developmental Psychology, 47(5), 1342-1352. doi:10.1037/a0024726.

Molina, B. S. G., \& Chassin, L. (1996). The parent-adolescent relationship at puberty: Hispanic ethnicity and parent alcoholism as moderators. Developmental Psychology, 32(4), 675-686. doi:10.1037//0012-1649.32.4.675.

Muthén, L. K., \& Muthén, B. O. (1998-2012). Mplus user's guide (7th Edn.). Los Angeles, CA: Muthén \& Muthén.

Ohannessian, C. M., Lerner, R. M., Lerner, J. V., \& von Eye, A. (2000). Adolescent-parent discrepancies in perceptions of family functioning and early adolescent self-competence. International Journal of Behavioral Development, 24(3), 362-372. doi:10. 1080/01650250050118358.

Pels, T., \& Nijsten, C. (2003). Myths and realities of diversity in parenting and parent-child relations: A comparison of indigenous and non-indigenous families in the Netherlands. In L. Hagendoorn., J. Veenman., \& W. Vollebergh (Eds.), Integrating immigrants in the Netherlands. Cultural versus socio-economic integration (pp. 63-90). Aldershot/Burlington, UK: Ashgate.

Pelton, J., \& Forehand, R. (2001). Discrepancy between mother and child perceptions of their relationship: I. consequences for adolescents considered within the context of parental divorce. Journal of Family Violence, 16(1), 1-15. doi:10.1023/A:1026527008239.

Pelton, J., Steele, R. G., Chance, M. W., \& Forehand, R. (2001). Discrepancy between mother and child perceptions of their relationship: II. consequences for children considered within the context of maternal physical illness. Journal of Family Violence, 16(1), 17-35. doi:10.1023/A:1026572325078.

Richardson, D. R., Hammock, G. S., Smith, S. M., Gardner, W., \& Signo, M. (1994). Empathy as a cognitive inhibitor of interpersonal aggression. Aggressive Behavior, 20(4), 275-289. doi:10.1002/ 1098-2337(1994)20:4<275::AID-AB2480200402>3.0.CO;2-4.

Shek, D. T. (1998). A longitudinal study of Hong Kong adolescents' and parents' perceptions of family functioning and well-being. The Journal of Genetic Psychology, 159(4), 389-403. doi:10. 1080/00221329809596160.

Sijtsma, K. (2009). On the use, the misuse, and the very limited usefulness of Cronbach's alpha. Psychometrika, 74(1), 107-120.

Sillars, A., Smith, T., \& Koerner, A. (2010). Misattributions contributing to empathic (in)accuracy during parent-adolescent conflict discussions. Journal of Social and Personal Relationships, 27(6), 727-747. doi:10.1177/0265407510373261.

Singer, T. (2006). The neuronal basis and ontogeny of empathy and mind reading: Review of literature and implications for future research. Neuroscience and Biobehavioral Reviews, 30(6), 855-863. doi:10.1016/j.neubiorev.2006.06.011.

Smetana, J. (1989). Adolescents' and parents' reasoning about actual family conflict. Child Development, 60(5), 1052-1067. http:// europepmc.org/abstract/MED/2805883.

Soenens, B., Park, S., Vansteenkiste, M., \& Mouratidis, A. (2012). Perceived parental psychological control and adolescent depressive experiences: A cross-cultural study with Belgian and southkorean adolescents. Journal of Adolescence, 35(2), 261-272. doi:10.1016/j.adolescence.2011.05.001.

Sonnby-Borgström, M. (2002). Automatic mimicry reactions as related to differences in emotional empathy. Scandinavian Journal of Psychology, 43(5), 433-443. doi:10.1111/14679450.00312

Statistics-Netherlands. (1993). Standaard beroepenclassificatie 1992 [standardized classification of occupations 1992]. Voorburg/ Heerlen: Netherlands Central Bureau of Statistics.

Steinberg, L. (1988). Reciprocal relation between parent-child distance and pubertal maturation. Developmental Psychology, 24(1), 122-128. doi:10.1037/0012-1649.24.1.122. 
Steinberg, L. (2001). We know some things: Parent-adolescent relationships in retrospect and prospect. Journal of Research on Adolescence, 11(1), 1-19. doi:10.1111/1532-7795.00001.

Stocks, E. L., Lishner, D. A., \& Decker, S. K. (2009). Altruism or psychological escape: Why does empathy promote prosocial behavior? European Journal of Social Psychology, 39(5), 649-665. doi:10.1002/ejsp.561.

Van der Graaff, J., Branje, S. J. T., De Wied, M., Hawk, S. T., Van Lier, P., \& Meeus, W. (2013). Perspective taking and empathic concern in adolescence: Gender differences in developmental changes. Developmental Psychology. doi:10.1037/a0034325.

Van Kleef, G. A., De Dreu, C. K. W., \& Manstead, A. S. R. (2004). The interpersonal effects of emotions in negotiations: A motivated information processing approach. Journal of Personality and Social Psychology, 87(4), 510-528. doi:10.1037/00223514.87.4.510.

Van Lier, P. A. C., Frijns, T., Neumann, A., Den Exter Blokland, E., Koot, H. M., \& Meeus, W. H. J. (2011). The RADAR young study: Design, description of sample, and validation of cohort assignment. Unpublished manuscript.

Van Lissa, C. J., Hawk, S. T., de Wied, M., Koot, H. M., van Lier, P., \& Meeus, W. (2014). The longitudinal interplay of affective and cognitive empathy within and between adolescents and mothers. Developmental Psychology, 50(4), 1219-1225. doi:10.1037/ a0035050.

Walton, J. W., Johnson, S. B., \& Algina, J. (1999). Mother and child perceptions of child anxiety: Effects of race, health status, and stress. Journal of Pediatric Psychology, 24(1), 29-39. doi:10. 1093/jpepsy/24.1.29.

Caspar J. Van Lissa, MSc is a PhD candidate at the Research Centre Adolescent Development, Utrecht University, The Netherlands. He received his cum laude research masters in social psychology from VU University, The Netherlands in 2010. His research interests include adolescent empathy development, adolescent-parent conflict resolution, and self-regulation.
Skyler T. Hawk, PhD is an assistant professor at the Chinese University of Hong Kong. He received his doctorate in social psychology from the University of Amsterdam, The Netherlands in 2010. His research interests include emotion expression, empathy, parent-child conflict resolution, and adolescent privacy and experiences of parental privacy invasion.

Susan J. T. Branje, PhD is a professor at the Research Centre Adolescent Development, Utrecht University. She received her cum laude doctorate in adolescent development from the Radboud University Nijmegen, The Netherlands in 2003 . Her work generally focuses on understanding the developmental changes in adolescents' relationships with parents, siblings, friends, and romantic partners and the associations with development of adolescent adjustment.

Hans M. Koot, PhD is a professor at the department of Developmental Psychology at VU University, Amsterdam, The Netherlands. He received his doctorate from the Erasmus University, Rotterdam, The Netherlands. His major research interest regard the etiology, diagnostics, and intervention of emotional and behavioral problems in children and adolescents.

Pol A. C. Van Lier, PhD a professor of Developmental Psychopathology at the Department of Developmental Psychology at the VU University in Amsterdam, The Netherlands. He received his doctorate from Erasmus University Rotterdam, The Netherlands. His research interests are in the area of development of psychopathology in children, with a particular focus on social and personal influences.

Wim H. J. Meeus, PhD is Professor of Adolescent Development at Utrecht University, and Professor of Developmental Psychology at Tilburg University, The Netherlands. He received his doctorate in Social Psychology from Utrecht University, The Netherlands. His major research interests include identity and personality development, personal relationships, and psychosocial problems in adolescence. 